\title{
INFLUENCIA DE LOS FACTORES AMBIENTALES EN EL NÚMERO DE INGRESOS POR URGENCIAS EN EL COMPLEJO HOSPITALARIO «JUAN CANALEJO» DE A CORUÑA: ELABORACIÓN DE UN MODELO DE PREDICCIÓN
}

\author{
M. ${ }^{a}$ Belén Lage Ferrón (1), Julio Díaz Jiménez (1) Juan Jesús Gestal Otero (2), M. ${ }^{\text {a }}$ de la Sierra \\ Pajares Ortíz (1) y Juan Carlos Alberdi Odriozola. \\ (1) Centro Universitario de Salud Pública de Madrid. \\ (2) Departamento de Medicina Preventiva. Universidad de Santiago.

\section{RESUMEN} \\ Fundamento: En este trabajo se trata de establecer, respec- \\ to al servicio de urgencias del hospital Juan Canalejo de A \\ Coruña, las posibles asociaciones entre el numero de ingresos \\ por causas orgánicas, cardiovasculares y respiratorias, y las \\ variables meteorológicas introducidas como variables exóge- \\ nas con el fin de elaborar un modelo de predicción.

\section{ABSTRACT \\ The Influence of Environmental Factors on the Number of Emergency Room Admissions at the Juan Canalejo Medical Center Complex in Corunna: Drafting a Prediction Model}

Métodos: Se utilizó la metodología de Box-Jenkins para obtener modelos ARIMA univariados de las series temporales consideradas. Se establecen funciones de correlación cruzada (FCC) entre las series de residuales que permitan establecer pesos y retrasos entre las variables. para una posterior modelización mediante modelos ARIMA multivariantes que incluyen variables ambientales. El periodo de tiempo estudiado fue de 1994 a 1996.

Resultados: Los ingresos urgentes por causas orgánicas aumentan significativamente entre 0 y 2 días después de un ascenso de la temperatura ambiental. Los ingresos por causas respiratorias se asocian con los descensos de temperalura con 10-14 retrasos, mientras que los ingresos por causas circulatorias se ven aumentados significativamente por el calor a largo plako (10 retrasos). En mayores de 65 años se registran. además, iumentos significitivos de los ingresos urgentes por causas circulatorias, en relación con el frio. a corto plazo. Los modelos ARIMA multivariantes que contemplan el efecto de variables ambientales ofrecieron un mejor ajuste para todas las variables de ingresos.

Conclusiones: El número de ingresos en el servicio de urgencias en el Complejo Hospitalario Juan Canalejo de A Coruña por causals orgánicas. respiratorias y circulatorias, presenta un patrón de comportamiento estacional. Los ingresos por causas respiratorias están asociados al descenso de la temperatura ambiental. mientras que los ingresos por causas circulatorias se ven afectados fundamentalmente por el calor, aunque también por el frío. en mayores de 65 años. Los modelos ARIMA multivariantes, incluyendo variables climatológicas. ofrecen un sistema de predicción de ingresos en función de dichas variables que puede ser útil desde el punto de vista de la gestión hospilabaria.

Palabras clave: Temperatura del aire. Series temporales. Ingresos hospitalarios. Modelos ARIMA.

\footnotetext{
Correspondencia:

Julio Díaz Jiménez.

Centro Universitario de Salud Pública.

C/ General Oraa, 39.

28006 Madrid

Corren electrónico: julio.diaz@ nam.es.
}

Background: This study is aimed at establishing the possible associations between the number of admissions through the emergency room at the «Juan Canalejol» Hospital in Corunna in 1994-1994 due to organic, circulatory and respiratory reasons and the weather variables introduced as being exogenous for the purpose of preparing a prediction model

Methods: The Box-Jenkins methodology is used for obtaining univariate ARIMA models of the time-based series taken into consideration. Cross-Correlation Functions ( $\mathrm{CCF}^{*}$ s) are established among the series of residuals which afford the possibility of establishing weights and lags among the variables for a subsequent modeling by means of multivariate ARIMA models which include environmental variables.

Results: The emergency admissions for organic reasons significantly increase 0-2 days following a rise in temperature. The admissions due to respiratory ailments are associated with drops in temperature with 10-14 lags, whilst the admissions for circulatory reasons increase significantly due to long-lasting spells of hot weather $(10$ lags). For people over age 65 . significant increases in emergency admissions for circulatory reasons are also recorded with cold snaps. The multivariate ARIMA models that take into account the effect of environmental variables provided the best adjustment for all of the admissions variables.

Conclusions: The number of emergency room adimissions at the "Juan Canalejo" Medical Center Complex in Corunna due to organic, respiratory and circulatory causes shows a seasonal behavior pattern. The admissions for respiratory reasons are associated with a drop in temperature, whilst the admissions for circulatory reasons are affected fundamentally by hot weather, although also by cold weather as regards people over age 65 . The multivariate ARIMA models including climate-related variables provide a system for predicting admissions in terms of said variables that can be useful from the standpoint of hospital management.

Key words: Air temperature. Time series. Hospital admissions. ARIMA models. 


\section{INTRODUCCIÓN}

La influencia que los factores meteorológicos, como la temperatura $\left(\mathrm{T}^{\mathrm{a}}\right.$ ) o la humedad relativa del aire (HU), ejercen sobre la salud es conocida desde la antiguiedad, si bien existe en la actualidad un creciente interés por estudiar los efectos en salud de los factores medioambientales. Así, las variaciones estacionales de la mortalidad constituyen un fenómeno ampliamente estudiado en distintos paises ${ }^{1,2}$, siendo bien conocido el pico de mortalidad invernal favorecido por las bajas temperaturas. En ocasiones, se detecta también un pico estival de menor amplitud, en lugares de climatología caracterizada por altas temperaturas ${ }^{3,4}$.

En general, son numerosos los estudios que sugieren que la temperatura ambiental y la mortalidad presentan una relación en forma de $\mathrm{V}$, si bien el mínimo de mortalidad se sitúa en uno u otro punto en función del clima propio de cada lugar ${ }^{3,5,6}$. En cuanto a las causas de muerte que muestran una mayor asociación con los cambios de temperatura se encuentran las enfermedades del aparato respiratorio y circulatorio ${ }^{7}$, siendo la sobremortalidad por bajas temperaturas en gran parte explicada por enfermedades de tipo respiratorio ${ }^{5.8}$.

Recientemente, un estudio realizado en la Comunidad de Madrid, encuentra una clara periodicidad de la mortalidad diaria, detectándose un pico invernal que afecta fundamentalmente a mujeres mayores de 65 años con enfermedades del aparato respiratorio y a varones mayores de 44 con afecciones del aparato circulatorio, respiratorio y digestivo ${ }^{9}$. El pico estival aparece relacionado con muertes por accidente cerebrovascular agudo en mujeres mayores de 65 años ${ }^{10,11}$.

Por otro lado, en los últimos años, se han realizado diferentes estudios epidemiológicos que se centran en los efectos de las variables ambientales sobre la morbilidad ${ }^{11-14}$. Así, en Atenas, diferentes autores pusieron de manifiesto los efectos severos que sobre la salud producían las condiciones de calor extremo. Por ello, propusieron la creación de un índice termohigrométrico basado en la temperatura máxima diaria y en la humedad relativa, útil para predecir los efectos en salud de determinadas condiciones meteorológicas ${ }^{15}$.

Estos estudios, que buscan una asociación entre los factores climáticos y los ingresos hospitalarios, se centran fundamentalmente en las enfermedades del aparato respiratorio, en particular en pacientes con EPOC ${ }^{16-18}$ y enfermedades del aparato circulatorio ${ }^{19}$.

Así, en base a la evidente asociación, entre factores meteorológicos y morbilidad por diferentes causas, encontrada por numerosos autores de diversos países, parece de interés estudiar el impacto que esta relación supone y comprobar si se traduce en un incremento del número de ingresos hospitalarios en una ciudad de características climatológicas menos extremas que las referidas en la mayoría de los estudios. Avanzar en este conocimiento supondría el inicio de un camino que conduciría a la posibilidad no sólo de predecir picos de ingresos y elaborar hipótesis causales sino, también, disponer de las medidas preventivas oportunas.

Se pretende así, por un lado, estudiar el efecto que sobre el número de ingresos hospitalarios ejercen diferentes variables atmosféricas. Para ello se modelizará el comportamiento del número de ingresos hospitalarios en el hospital Juan Canalejo de A Coruña por urgencias, por distintas causas, partiendo de datos de ingresos no programados durante el período 19941996. En segundo lugar, se obtendrán modelos multivariantes que permitan conocer el comportamiento de las series de ingresos en función de variables medioambientales y controlando por diferentes factores de confusión.

\section{MATERIAI, Y MÉTODOS}

La población objeto de estudio fue la procedente del Área Sanitaria de A Coruña, integrada por 36 municipios, siendo la zona más densamente poblada la propia ciudad de 
A Coruña, con cerca de 250000 habitantes. El periodo de tiempo considerado fue de 3 años, desde enero de 1994 hasta diciembre de 1996.

\section{Datos de ingresos hospitalarios:}

Los datos relativos al número de ingresos hospitalarios por urgencias, fueron obtenidos a partir de la base de datos CMBD (conjunto mínimo de datos básicos) del hospital Juan Canalejo de A Coruña. Los ingresos diarios fueron clasificados en función de las causas de ingreso (diagnóstico principal) codificada según la lista detallada de tres dígitos de la CIE-9. La clasificación se realizó para el total de casos y para mayores de 65 años:

- Ingresos debidos a causas orgánicas (1799).

- Ingresos por enfermedades del aparato respiratorio (460-519).

- Ingresos por enfermedad circulatoria (390-459).

\section{Datos medioambientales}

Fueron facilitados por el Instituto Nacional de Meteorología (Centro Meteorológico de A Coruña). Los registros diarios durante el período considerado corresponden a las siguientes variables: Temperatura (máxima, mínima y media); humedad relativa medida a las 7 horas $(\mathrm{Hu} 7 \mathrm{~h})$; velocidad del viento expresada como «recorrido», que representa los kilómetros recorridos por el viento desde las 0 horas hasta las 24 .

La ubicación de la estación medidora fue en el casco urbano de A Coruña, por ser la de mayor fiabilidad y la que mejor representaba los valores de exposición de la mayor parte de la población de referencia.

\section{Otras variables}

Con el fin de controlar posibles factores de confusión a la hora de obtener los modelos multivariante, se crearon las variables:
- «Gripe», debido a su posible influencia en la aparición de picos en las series de ingresos por afecciones del aparato respiratorio. Los datos del número de casos semanales de gripe en el área sanitaria de estudio fueron codificados como variable dicotómica, según la existencia o no de epidemia de gripe, proporcionados por el Servicio de Epidemiología de la Delegación Provincial de Sanidad de A Coruña.

— «Descargas de haba de soja» en el puerto de A Coruña durante el período de tiempo considerado, variable destinada a controlar el posible efecto que las grandes descargas de soja producen en individuos alérgicos $\mathrm{y}$, por tanto, en el agravamiento de detcrminados procesos respiratorios. Dichos datos fueron obtenidos a través de la autoridad portuaria de A Coruña.

- Codificación: es una variable ficticia que se ha creado debido a que la codificación en el periodo de estudio no ha sido constante, variando del $93 \%$ de ingresos codificados en febrero del 94 hasta lograr el $100 \%$ en enero del 96. El valor de esta variable lo constituyen el porcentaje de ingresos urgentes codificados cada mes.

- Día de la semana: se obtuvo, como consecuencia de la distribución no homogénea de las frecuencias de ingresos por día de la semana. La variable toma valores desde 1 (domingo) hasta 7 (lunes).

- Tendencia: Se crea esta variable para controlar la pendiente positiva de las series de ingresos, tomando el valor del número de día cntrc 1 y 1086.

Además se crearon también las correspondientes variables retrasadas de las variables ambientales asociadas significativamente, con las variables de ingresos.

Se realizaron diagramas de dispersión para establecer la relación funcional entre las diferentes variables ambientales y los ingresos hospitalarios. La temperatura mostró una distribución en forma de $\mathrm{V}$, con una tempe- 
ratura máxima de ingresos mínimos en 22 ${ }^{\circ} \mathrm{C}$ para el caso de los ingresos por todas las causas. Es claro que habrá una temperatura de mínimos ingresos para cada causa específica considerada y para cada grupo de edad. No obstante, las diferencias obtenidas entre estas temperaturas apenas llegan a las décimas de grado. Esto llevó a que, por simplicidad, la temperatura se dividiera en las variables temperatura cálida (tcal) y temperatura fría (tfrío), en función de su separación de la temperatura de confort (la de mínimos ingresos) de $22{ }^{\circ} \mathrm{C}$.

Para evaluar la influencia de las variables ambientales sobre el número de ingresos por urgencias por distintas causas, se modelizó cada una de las series temporales correspondientes, empleando modelos autorregresivos integrados y de media móvil (ARIMA) de Box-Jenkins ${ }^{20,21}$, en función de la propia historia de las series. Para ello, fueron identificadas previamente las periodicidades de cada serie mediante el análisis espectral y calculadas las funciones de autocorrelación simple (ACF) y parcial (PACF) de cada una de las series. Así, los modelos ARIMA univariados obtenidos para cada una de las variables de ingresos hospitalarios, representan el comportamiento de cada variable, teniendo en cuenta únicamente la historia de la serie. Estos modelos solamente permitirán predecir situaciones episódicas de ingresos que hayan ocurrido con anterioridad.

Tras la obtención de cada uno de los modelos para las variables de ingresos y variables ambientales, se realizó el cruce de cada una de estas últimas con cada una de las seis series de ingresos, mediante el método de problanqueo de Box-Jenkins, obteniendo las diferentes funciones de correlación cruzada (FCC) de las series residuales. Además, con el fin de aumentar la precisión en las estimaciones, se consideraron tres períodos de tiempo: todo el año, invierno (meses de noviembre a marzo) y verano (meses de junio a agosto). Los valores estadísticamente significativos que muestren asimetría en la distribución de la
FCC entre los retrasos positivos y negativos permitirán inferir una relación estadísticamente significativa entre ambas variables, pudiéndose identificar pesos y retrasos significativos entre ellas.

En base a las asociaciones significativas en los retardos que marquen las funciones de correlación cruzada, se identificaron las variables ambientales con influencia sobre las variables de ingresos, creándose las correspondientes variables retrasadas, necesarias para la obtención de los modelos multivariantes de las series de ingresos. Cada modelo incluyó las distintas variables ambientales objeto de estudio junto con las creadas, para controlar posibles factores de confusión. Entre estas se encuentran la variable relativa al porcentaje de casos codificados, la variable día de la semana, la variable tendencia y las variables dicotómicas «gripe» $\mathrm{y}$ «descargas de haba de soja». Las estacionalidades de mayor periodo fueron consideradas introduciendo funciones sinusoidales de periodo un año, 6 meses y 3 meses.

La bondad de los ajustes de cada modelo fue evaluada analizando la estructura de ruido blanco de los residuos, varianza mínima de los mismos y el mayor coeficiente de correlación entre el ajuste del modelo y el valor real de la serie modelizada. La estabilidad de los estimadores del modelo se confirmó empleando el método Jack-knife, que consiste en repetir el modelo multivariante omitiendo uno a uno los años comprendidos en el periodo de estudio, con el fin de controlar de este modo las posibles variaciones de los estimadores respecto a la estacionalidad anual. El análisis estadístico de los datos fue realizado empleando el paquete informático SPSS para Windows.

\section{RESULTADOS}

La tabla 1 muestra la distribución del número de ingresos diarios por urgencias en el hospital Juan Canalejo de A Coruña, por causas orgánicas, respiratorias y circulatorias, tanto para el total de casos, como para hombres, mujeres y mayores de 65 años. La 
Tabla 1

Media y desviación estándar de las variables analizadas

\begin{tabular}{|c|c|c|c|c|}
\hline Causas de ingrese & Total & Hombres & Mujeres & $>65$ años \\
\hline ORGANICAS & $37.7 \pm 8.9$ & $20,9 \pm 5,8$ & $16.7 \pm 4.9$ & $15.5 \pm 5.1$ \\
\hline RESPIRATORIAS & $5,9 \pm 3,4$ & $3,8 \pm 2,4$ & $2.1 \pm 1,7$ & $2.3 \pm 1.8$ \\
\hline CIRCULATORIAS & $9,2 \pm 3,5$ & $5,5 \pm 2,5$ & $3,7 \pm 2.1$ & $5,8 \pm 2,7$ \\
\hline \multicolumn{5}{|l|}{ Variables atmosféricas: } \\
\hline Temperatura mínima $\left({ }^{\circ} \mathrm{C}\right)$ & \multicolumn{4}{|c|}{$11,9 \pm 3,4$} \\
\hline Temperatura media $\left({ }^{\circ} \mathrm{C}\right)$ & \multicolumn{4}{|c|}{$14.9 \pm 3,6$} \\
\hline Temperatura máxima $\left({ }^{\circ} \mathrm{C}\right)$ & \multicolumn{4}{|c|}{$17,9 \pm 4,1$} \\
\hline Racha máxima $(\mathrm{Km} / \mathrm{h})$ & \multicolumn{4}{|c|}{$39,1 \pm 14,9$} \\
\hline Recorrido $(\mathrm{Km})$ & \multicolumn{4}{|c|}{$292.5 \pm 132.4$} \\
\hline $\mathrm{Hu} 7 \mathrm{~h}(\%)$ & \multicolumn{4}{|c|}{$81,0 \pm 9,5$} \\
\hline
\end{tabular}

media de ingresos por distintas causas fue de 37,7 , siendo siempre mayor para el grupo de hombres que para el de mujercs. El número de ingresos en los mayores de 65 años representa algo menos de la mitad del total de ingresos, salvo para causas circulatorias. Se observa también la media y desviación estándar de las variables ambientales pu- diendo apreciarse la ausencia de temperaturas extremas así como los altos niveles de humedad relativa.

La evolución temporal de las series de ingresos urgentes para el total de casos, y de las temperaturas máxima y mínima pueden verse en las figuras 1 y 2 respectiva-

Figura 1

Series de ingresos por urgencias. 1994-1996. Todos los casos

Evolución temporal de los Ingresos por Urgencias en el Hospital Juan Canalejo de A Coruña por causas orgánicas, respiratorias y circulatorias (1994-1996).

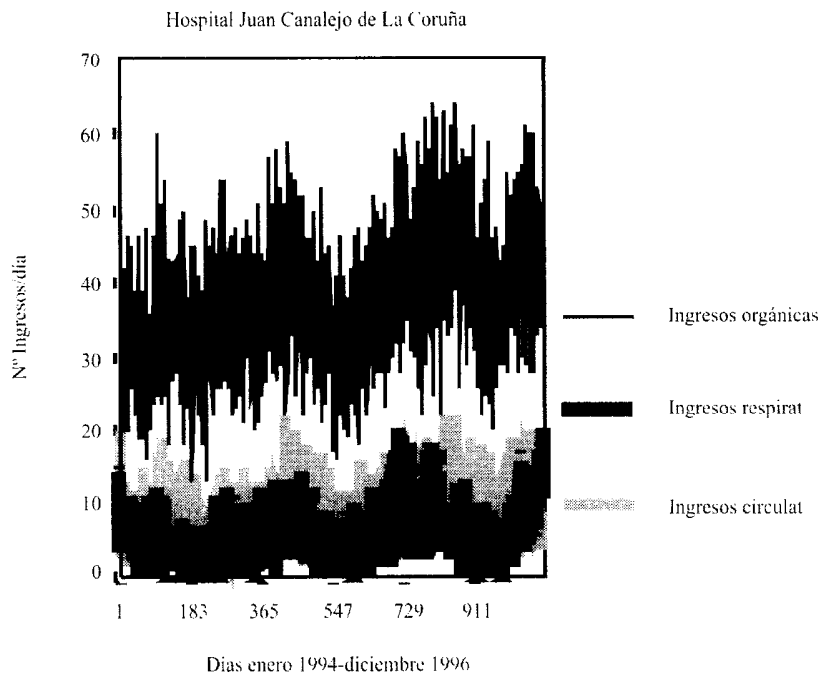




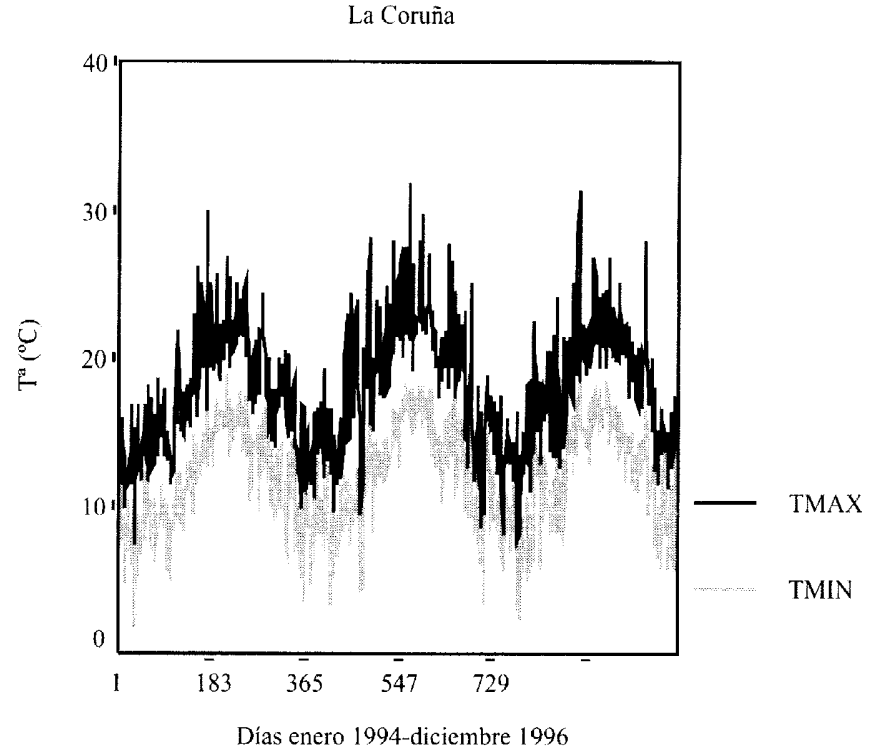

mente. El comportamiento estacional aparece claramente reflejado en las series de temperatura, no siendo tan evidente en las de ingresos.

La distribución de la varianza en función de las frecuencias permitió identificar la existencia de tendencias y periodicidades significativas al $99 \%$ para cada una de las variables de estudio, como se resumen en la tabla 2. Las variables ambientales de temperatura y humedad relativa mostraron un comportamiento similar (ciclo anual y de 3 días) a diferencia de la variable relativa al viento ( 2 días). Las series de ingresos mostraron una tendencia positiva, así como periodicidades anual, semestral o trimestral, y de 7 ó 3 días. En la figuras 3 y 4 pueden verse dos ejemplos de representación gráfica del espectro de frecuencias frente a la densidad espectral.
Los modelos ARIMA obtenidos de cada una de las series (tabla 3 ), representan el comportamiento temporal de cada variable cn función de la propia historia de la serie. Para los ingresos totales por causas orgánicas el modelo ARIMA univariante fue de $(1,1,1)(0,1,1) 7$, lo que significa que tiene un componente autorregresivo (AR) de orden 1 una parte integrada (I) de 1 , y una media móvil (MA) de orden 1. Los componentes ARIMA de su parte estacional son 0,1 y 1 respectivamente, mostrando además una periodicidad de 7 días. De ello se deduce que los ingresos por causas orgánicas dependen del número de ingresos del día anterior $(A R=1)$. La tendencia de la serie también es recogida por el modelo $(\mathrm{I}=1)$ y su componente de aleatoriedad se refleja en su media móvil de 1. Para el grupo de mayores de 65 años el modelo fue similar pero mostrando un mayor componente aleatorio $(\mathrm{MA}=2)$, 
Tabla 2

Tendencias y periodicidades

\begin{tabular}{|c|c|c|c|}
\hline & & Tendencia & Periodicidades \\
\hline \multicolumn{4}{|l|}{ Variables dependientes } \\
\hline \multirow{3}{*}{ INGRESOS TOTALES } & ORG $\Lambda$ NICAS & SI $(+)$ & anual, semestral, 3días, 7 días \\
\hline & RESPIRATORIAS & SI $(+)$ & anual, semestral, 3 días \\
\hline & CIRCULATORIAS & SI $(+)$ & anual, semestral. 7 y 3 días \\
\hline \multirow{3}{*}{ INGRESOS MAYORES 65 AÑOS } & ORGÁNICAS & SI $(+)$ & anual, semestral, 7 días 3 días \\
\hline & RESPIRATORIAS & SI $(+)$ & anual, trimestral, 3 días \\
\hline & CIRCULATORIAS & SI $(+)$ & anual, semestral, 7 días \\
\hline \multicolumn{4}{|l|}{ Variables independientes } \\
\hline T. ${ }^{\text {a }}$ máxima $\left({ }^{\circ} \mathrm{C}\right)$ & & NO & anual, 3 días \\
\hline $\mathrm{T} .{ }^{a}$ mínima $\left({ }^{\circ} \mathrm{C}\right)$ & & NO & anual, 3 días \\
\hline $\mathrm{T},{ }^{a}$ media $\left({ }^{\circ} \mathrm{C}\right)$ & & NO & anual, 3 días \\
\hline Humedad relativa a las $7 \mathrm{~h}(\%)$ & & NO & anual, 3 días \\
\hline Recorrido viento $(\mathrm{Km})$ & & NO & 2 días \\
\hline
\end{tabular}

Figura 3

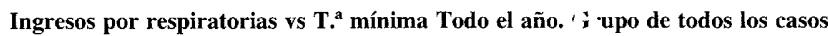

Espectro de potencia de la serie de temperatura máxima. Obsérvese para : frecuencia de 0,0026 la periodicidad anual significativa al $99 \%$

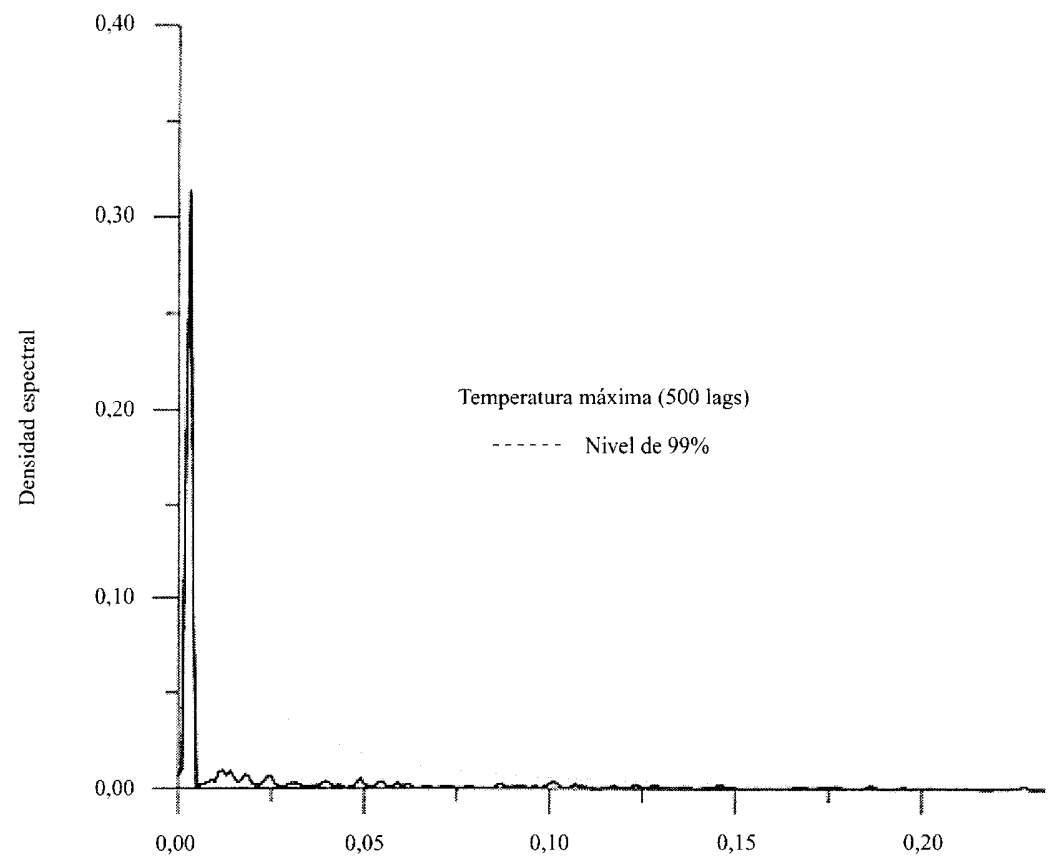

Frecuencias 
Figura 4

Admisiones diarias por urgencias por causas respiratorias

Espectro de frecuencias de la serie de ingresos por causas circulatorias en el que se observa una marcada tendencia en el lag 0 y periodicidades de 7 días y 3 días para las frecuencias 0,142 y 0,33 respectivamente.

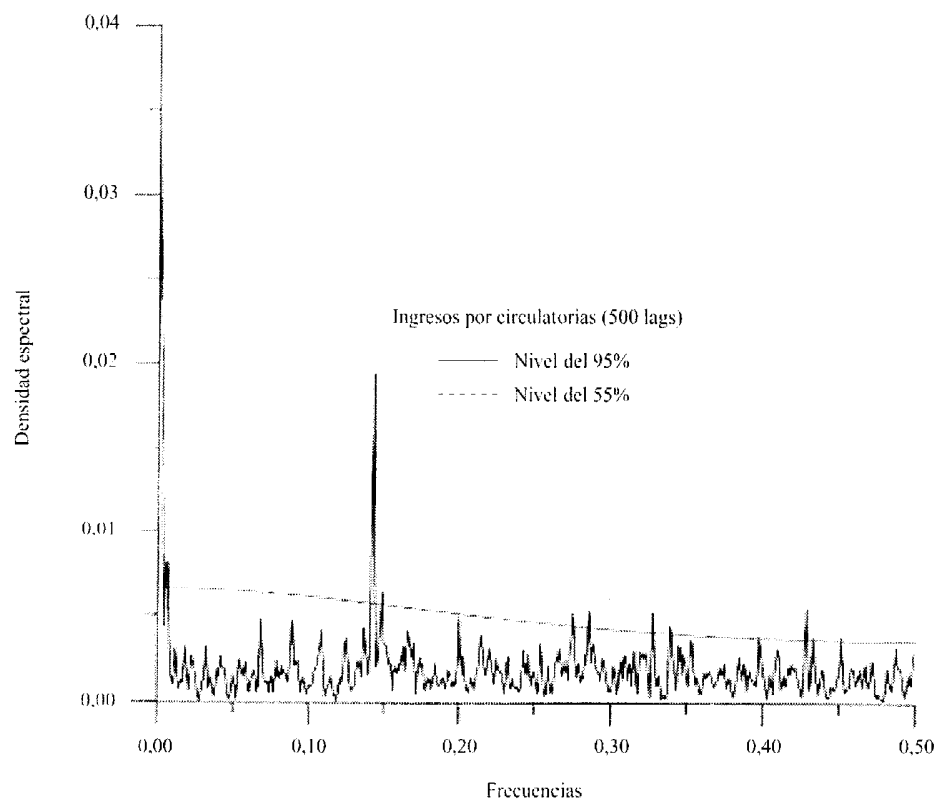

probablemente atribuible al menor número de casos registrados en este grupo.

Los demás modelos (tabla 3), admiten también funciones periódicas de período 1 año, 6 meses o 3 meses, que representan las estacionalidades. Cabe destacar cómo únicamente en los modelos de ingresos por respiratorias aparece periodicidad trimestral, mientras que las causas circulatorias presentan la semestral en concordancia con los resultados del análisis espectral.

En cuanto a los modelos ARIMA de las variables ambientales, las 3 series de temperatura presentan idéntico comportamiento, siendo diferente para la humedad relativa y el recorrido del viento. Todos los modelos de dichas variables, recogen la estacionalidad anual y la periodicidad de 3 días, así como la parte integrada de 0 que revela la inexistencia de tendencia (tabla 3 ).

Las FCC pusieron de manifiesto, como se muestra en la tabla 4, la asociación de ciertas variables de ingresos con la temperatura mínima y la temperatura máxima (figura 5), y también con el porcentaje de humedad relativa en el caso de ingresos invernales por causas respiratorias. Cabe destacar la influencia del descenso de la temperatura en el aumento del número de ingresos por causas respiratorias, fundamentalmente en invierno, con un retraso de 11-14 días (efecto de frío a largo plazo), como muestra la figura 6 . Los ingresos por causas respiratorias aumentan, además, durante el invierno el mismo día que la temperatura aumenta y desciende la humedad relativa (días calurosos de invierno). En cuanto a la variable relativa al vicnto, no se asoció significativamente con ninguna de las causas de ingreso. 
Tabla 3

Modelos ARIMA univariados

\begin{tabular}{|c|c|c|}
\hline & & MODELOS ARIMA \\
\hline \multicolumn{3}{|l|}{ Variables dependientes } \\
\hline & ORGÁNICAS & $(1,1,1)(0,1,1) 7$ \\
\hline INGRESOS TOTALES & RESPIRATORIAS & $(0,1,1)(1,1,2) 3+$ SENO +COSENO + COSTRI \\
\hline & CIRCULATORIAS & $(0,1,1)(0,1,1) 7+$ SENO + COSENO + SENSEM \\
\hline \multirow{3}{*}{ INGRESOS MAYORES 65 AÑOS } & ORGÁNICAS & $(1,1,2)(0,1,1) 7$ \\
\hline & RESPIRATORIAS & $(0,1,1)(0,1,1) 3+$ COSTRI \\
\hline & CIRCULATORIAS & $(0,1,1)(0,1,1) 7+$ SENO+ COSENO+ SENSEM \\
\hline \multicolumn{3}{|l|}{ Variables independientes } \\
\hline T. ${ }^{\text {a }}$ máxima $\left({ }^{\circ} \mathrm{C}\right)$ & & $(2,0,1)(1,0,1) 3+$ SENO + COSENO \\
\hline $\mathrm{T} .{ }^{a}$ mínima $\left({ }^{\circ} \mathrm{C}\right)$ & & $(2,0,1)(1,0,1) 3+$ SENO + COSENO \\
\hline T..$^{a}$ media $\left({ }^{\circ} \mathrm{C}\right)$ & & $(2,0,1)(1,0,1) 3+$ SENO + COSENO \\
\hline Humedad relativa a las $7 \mathrm{~h}(\%)$ & & $(1,0,0)(2,0,1) 3+$ SENO + COSENO \\
\hline Recorrido viento $(\mathrm{Km})$ & & $(2,0,0)(1,0,1) 3+$ SENO + COSENO \\
\hline
\end{tabular}

SENO: Función seno de perindicidad anual; COSENO: Función coseno de periodicidad anual; SENSEM: Función seno de periodicidad semestral; COSTRI: Función coseno de periodicidad trimestral.

En esta misma tabla se analizan las asociaciones detectadas para el grupo de mayores de 65 años para todas las causas de ingreso analizadas, siendo, en general, similares a las descritas para el grupo de todas las edades, excepto para los ingresos por causas circulatorias. En este caso el número de ingresos se ve aumentado por un efecto de calor en verano (con 7-15 días de retraso) y por un efecto de frío a corto plazo en invierno (1-2 días de retraso).

La elaboración de los correspondientes modelos multivariantes para cada una de las series permite la predicción de su comportamiento en función de las variables ambien-

Tabla 4

Retardos en los que se establecen asociaciones significativas en las FCC

Grupo de todos los casos

\begin{tabular}{|lccccccccc|}
\hline & \multicolumn{3}{c}{ Orgánicas } & \multicolumn{3}{c}{ Respiratorias } & \multicolumn{2}{c|}{ Circulatorias } \\
\cline { 2 - 9 } & Año & Invierno & Verano & Año & Invierno & Verano & Año & Invierno & Verano \\
\hline T. MAX. $\left({ }^{\circ} \mathrm{C}\right)$ & - & $2(+)$ & $0(+)$ & 10 a $14(-)$ & 10 a $14(-)$ & - & $10(+)$ & $10(+)$ & - \\
T. MIN. $\left({ }^{\circ} \mathrm{C}\right)$ & - & 0 y $2(+)$ & - & 10 a $14(-)$ & $0(+)$ & 10 a $14(-)$ & - & - & - \\
& & & - & - & $0(+)$ & $0(+)$ & & & - \\
HU7H $(\%)$ & - & - & - & - & $0(-)$ & - & - & - \\
RECORR & - & - & - & - & - & - & - & - \\
\hline
\end{tabular}

Grupo de mayores de 65 años

\begin{tabular}{|lccccccccc|}
\hline & \multicolumn{3}{c}{ Orgánicas } & \multicolumn{3}{c}{ Respiratorias } & \multicolumn{2}{c|}{ Circulatorias } \\
\cline { 2 - 8 } & Año & Invierno & Verano & Año & Invierno & Verano & Año & Invierno & Verano \\
\hline T. MAX & - & 1 a $3(+)$ & - & 10 a $14(-)$ & 10 a $14(-)$ & - & $2(-)$ & - & - \\
& & & & & $3(+)$ & & & & \\
T. MIN. & - & $2(+)$ & - & 10 a $14(-)$ & 10 a $14(-)$ & - & - & $1(-)$ & 7 y $15(+)$ \\
HU7H & - & - & - & - & - & - & - & - & - \\
RECORR & - & - & - & - & - & - & - & - & - \\
\hline
\end{tabular}


Figura 5

Funciones de correlación cruzada entre los ingresos por todas las causas y la temperatura máxima, seleccionados los meses de verano después del preblanqueo.

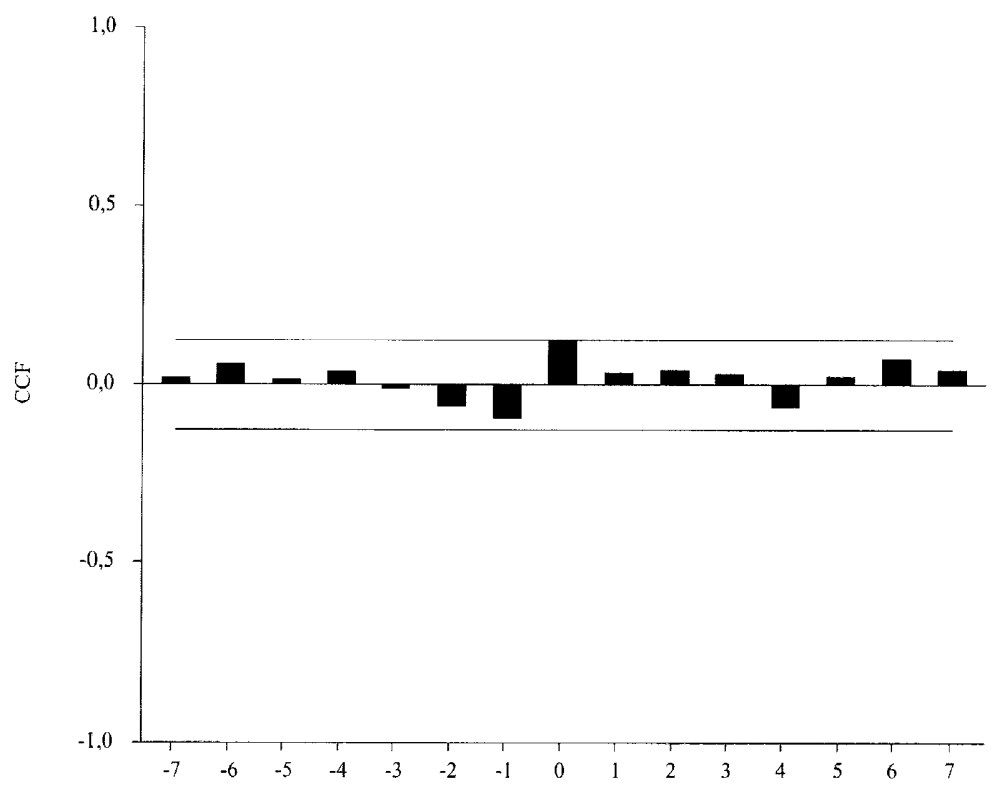

Figura 6

Funciones de correlación cruzada entre los ingresos por respiratorias y la temperatura mínima para todo el año.

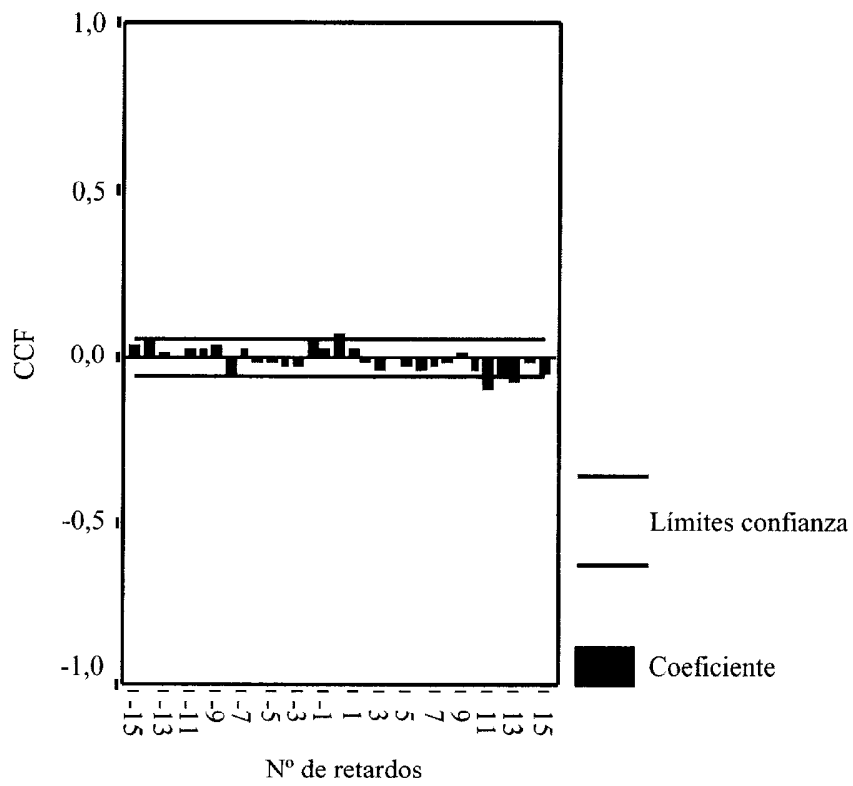


tales de influencia. Para ello la variable de mayor efecto, la temperatura, fue incluida en los modelos transformada en las variables: temperatura cálida (tcal) y temperatura fría (tfrío), definidas en el apartado de metodología. Los coeficientes de asociación para los diferentes modelos multivariantes obtenidos, para una $\mathrm{p}<0,05$, aparecen resumidos en la tabla 5. Dichos modelos únicamente recogen la variable ambiental de mayor influencia que fue la tfrío, siendo su efecto más patente en los ingresos por causas respiratorias en los mayores de 65 años. Así, los coeficientes obtenidos para este caso, determinan el aumento en ingresos/diarios por enfermedades respiratorias, que cabría esperara por cada grado centígrado que descienda la temperatura máxima por debajo de los $22{ }^{\circ} \mathrm{C}$. Esto supone un aumento entre 7 y 8 ancianos/día para una temperatura máxima de $12{ }^{\circ} \mathrm{C}$, con un retraso de 10 y 13 días, respectivamente.

Tabla 5

Coeficientes de asociación de diferentes variables externas frente a las distintas causas de ingreso en un modelo multivariante

\begin{tabular}{|c|c|c|c|c|c|c|}
\hline \multirow{2}{*}{ Variables } & \multicolumn{3}{|c|}{ Todos } & \multicolumn{3}{|c|}{ Mayores de 65 años } \\
\hline & Organ. & Resp. & Circ. & Organ. & Resp. & Circ. \\
\hline $\mathrm{n} 1$ & 0.009 & 0.004 & 0.002 & 0.004 & 0.001 & 0.001 \\
\hline \multirow[t]{6}{*}{ día } & $6.32(2)$ & & $1.07(2)$ & $1.72(2)$ & & $0.52(2)$ \\
\hline & $12.48(3)$ & $0.59(3)$ & $3.26(3)$ & $5.49(3)$ & $0.43(3)$ & $2.09(3)$ \\
\hline & $9.86(4)$ & $0.66(4)$ & $2.42(4)$ & $4.28(4)$ & $0.47(4)$ & $1.61(4)$ \\
\hline & $8.86(5)$ & $2.31(5)$ & $4.03(5)$ & $0.31(5)$ & $1.57(5)$ & \\
\hline & $7.23(6)$ & $1.54(6)$ & $3.23(6)$ & $0.36(6)$ & $0.95(6)$ & \\
\hline & $4.59(7)$ & $1.35(7)$ & $2.70(7)$ & $0.40(7)$ & $1.00(7)$ & \\
\hline codificación & 53.27 & - & 17.13 & 35.84 & - & 12.97 \\
\hline gripe & - & - & - & 0.575 & - & - \\
\hline \multirow[t]{2}{*}{ Tfrio } & $0.22(4)$ & $0.12(11)$ & $0.12(0)$ & $0.22(12)$ & $0.86(10)$ & $0.10(2)$ \\
\hline & $0.37(13)$ & $0.14(13)$ & & & $0.71(13)$ & \\
\hline
\end{tabular}

La figura 7 muestra la representación gráfica de la serie de ingresos por causas respiratorias, junto con sus correspondientes modelos univariado y multivariado. El análisis de los residuales muestra un ajuste ligeramente superior del modelo multivariante frente al modelo que únicamente contempla la historia de la serie.

\section{DISCUSIÓN}

La tendencia positiva de las series de ingresos es atribuible, por un lado, a que el porcentaje de casos codificados y, por tanto, considerados en el estudio, ha ido aumentando a lo largo del tiempo. En segundo lugar, la reapertura del hospital de Oza a principios de 1996, perteneciente al complejo hospitalario Juan Canalejo, repercute en el número de camas disponibles y, por tanto, en el número de admisiones por urgencias.

Las estacionalidades o periodicidades análogas entre las series de variables dependientes e independientes para 1 año y 3 días, nos llevan a la necesidad de modelizar cada una de las series, con el fin de conocer su comportamiento. Ello nos permitirá, tras el correspondiente filtrado de las series, comparar variables y establecer las correspondientes asociaciones una vez eliminadas las autocorrelaciones y estacionalidades análogas. La estacionalidad anual tiene una evidente explicación. La de tres días se relaciona con el paso de borrascas por nuestras la- 
Figura 7

Representación del modelo univariado y multivariado respecto a la serie de ingresos por causas respiratorias.

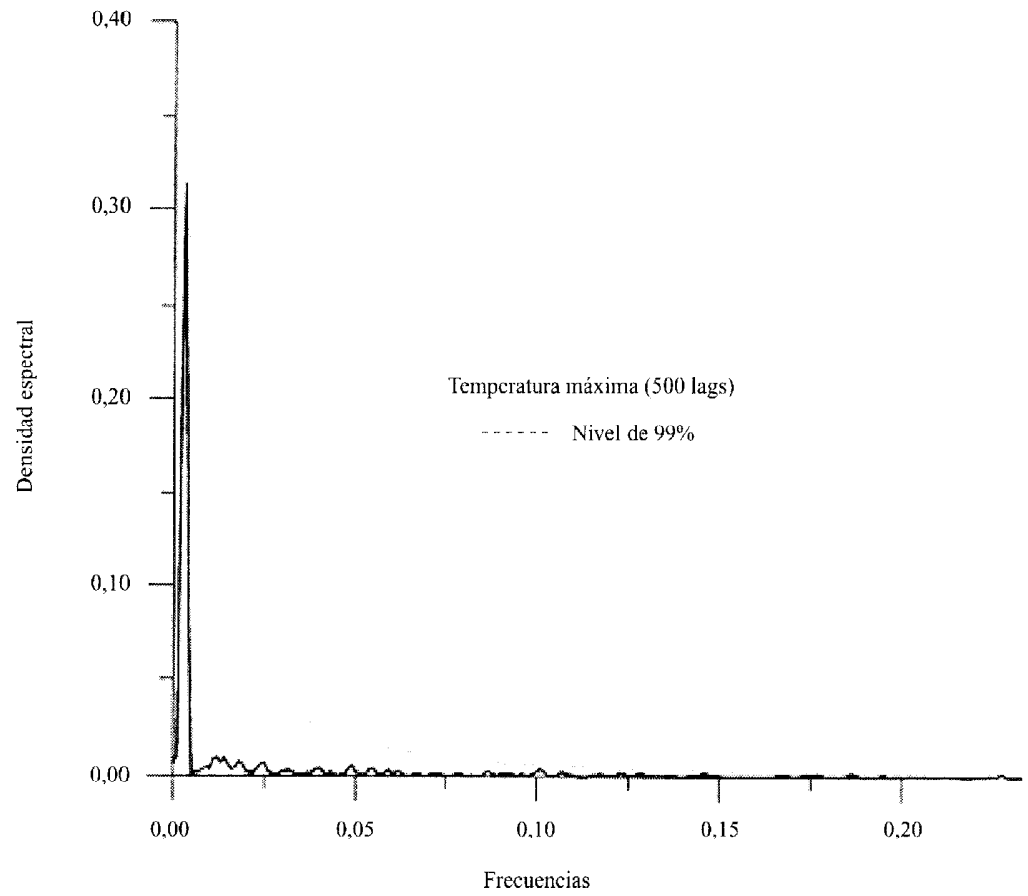

titudes, que influyen tanto en variaciones de variables meteorológicas como de otras de contaminación que no se contemplan en este estudio ${ }^{22}$.

Al estudiar la relación funcional entre las variables ingresos por causas orgánicas y temperatura máxima, obtuvimos una gráfica que se aproxima en cierta medida a la relación en $\mathrm{V}$ entre la mortalidad y la temperatura ya citada por numerosos autores ${ }^{5.8}$, si bien en el caso de A Coruña este valor es inferior al detectado en otros estudios ${ }^{3,10,11}$.

Con respecto a la influencia de las variables ambientales sobre las variables de ingresos, estudiada mediante las funciones de correlación cruzada (tabla 4), encontramos que para el grupo de lodos los casos, los ingresos por causas circulatorias se ven aumentados 10 días después de que aumente la temperatura máxima. Aparece, así, un efecto del calor sobre las enfermedades del aparato circulatorio, si bien se produce a más largo plazo de lo que ocurre en otros lugares, como la Comunidad de Madrid, en la cual el efecto del calor sobre la mortalidad por causas circulatorias se produce entre $\begin{array}{llll}0 & \text { y } & 3\end{array}$ días ${ }^{10}$.

Por otra parte, el número de ingresos por causas orgánicas en invierno, aumenta significativamente hasta 2 días después de un aumento de la temperatura máxima o mínima. Este aumento de ingresos en invierno asociado con situaciones de aumento de temperatura, puede estar en relación con la peculiar situación climatológica creada los días invernales en los que sopla viento del sur, que suele llevar temperaturas más elevadas a las normales en esa época del año ${ }^{23,24}$.

En cuanto a los ingresos por causas respiratorias, sufren un aumento significativo en- 
tre 11 y 14 días después de un descenso de la temperatura máxima o mínima, fundamentalmente en invierno. Esta asociación fue ya señalada por diferentes autores que encuentran un aumento significativo de la incidencia de reagudizaciones de procesos respiratorios al disminuir la temperatura y al aumentar la humedad relativa ${ }^{25}$. Este efecto del frío a largo plazo, coincide además con los resultados obtenidos en estudios que relacionan la temperatura y mortalidad por enfermedades respiratorias $3,10,11$. Otros trabajos, ponen de manifiesto que el efecto del frío sobre la mortalidad por enfermedades respiratorias se mantiene significativo hasta 15 días tras la exposición ${ }^{5}$, al igual que muestran las funciones de correlación cruzada obtenidas en este trabajo.

En los ingresos por respiratorias aparece de nuevo un efecto de calor invernal, el mismo día que la temperatura mínima aumenta y la humedad relativa desciende atribuible también a esas peculiaridades atmosféricas de los días calurosos de invierno.

En el grupo de personas mayores de 65 años, los resultados obtenidos fueron similares, salvo para los ingresos por enfermedades circulatorias. Así, los ingresos aumentan tras 1 ó 2 días después de una bajada de temperatura, fundamentalmente en invierno, mientras que en verano se produce un pico de ingresos a los 7 y otro a los 15 días de un aumento de la temperatura. Aquí surge un efecto de frío a corto plazo y un efecto de calor más a largo plazo. No aparecen resultados similares en este sentido en la bibliografía, sin embargo, estudios realizados en Escocia demuestran un claro comportamiento estacional de las enfermedades coronarias, apareciendo picos de ingresos en verano/invierno ${ }^{19}$. Otros autores encuentran también relación entre las temperaturas extremas y la mortalidad por enfermedades coronarias en ancianos, atribuible, al parecer, a un peor mecanismo de termorregulación ${ }^{26}$.

En cuanto a los modelos multivariantes obtenidos, nos permiten conocer la asocia- ción que existe entre el número de ingresos por distintas causas y diferentes variables, tanto ambientales, como la temperatura, como las introducidas para controlar factores de confusión. Así, la única variable ambiental que se mostró significativa en los modelos multivariantes fue la tfrio (variable que representa los grados centígrados por debajo de la temperatura de confort).

A la vista de los resultados obtenidos (tabla 5) se deduce, para el grupo de todos los casos, que por cada grado que la temperatura máxima esté por debajo de la de confort $\left(22^{\circ} \mathrm{C}\right)$, debemos añadir (al número de ingresos por causas orgánicas a $\operatorname{los} 22^{\circ} \mathrm{C}$ ), 0,22 ingresos más por causas orgánicas para el lag 4 y 0,37 para el lag 13. Por tanto, los días en que la temperatura máxima sea de $12{ }^{\circ} \mathrm{C}$, sabremos que 4 días después se producirá un aumento de 2,2 ingresos y que 13 días después se producirá un incremento de 3,7 ingresos por causas orgánicas sobre el valor mínimo.

Un estudio similar realizado en la Comunidad de Madrid ${ }^{27}$, muestra un coeficiente para el frío de 0,26 ingresos más por cada grado por debajo de la temperatura de confort de Madrid $\left(32^{\circ} \mathrm{C}\right.$ ). Aparece sin embargo un fuerte efecto del calor (coeficiente de 1,1) que no se aprecia en nuestro estudio. No es extraño la no aparición de dicho efecto del calor si tenemos en cuenta que durante 3 años no se ha superado la temperatura de $32{ }^{\circ} \mathrm{C}$ en la ciudad de A Coruña, temperatura a partir de la cual se han encontrado efectos significativos en Madrid.

El efecto del frío fue también significativo para las demás causas de ingreso, siendo especialmente acusado en el grupo de mayores de 65 años que ingresaban por causas respiratorias $(0,86-0,71$ con $10-13$ días de retraso).

Otras variables atmosféricas que resultaron estar estadísticamente asociadas a las variables dependientes durante la realización de las funciones de correlación cruzada, no se mostraron significativas en los 
modelos multivariantes. Sin embargo, los modelos multivariantes sí recogieron las variables más significativas en las FCC y en los mismos retrasos, mostrando de nuevo el efecto del frío a largo plazo para los ingresos por causas respiratorias. No pudo apreciarse, sin embargo, el efecto del calor que sí aparecía en algunas FCC. Esta diferencia puede ser atribuida a que las FCC fueron analizadas considerando los períodos de todo el año, invierno y verano, operación que no fue realizada en los modelos multivariantes. Por otra parte, hay que considerar que las FCC únicamente comparan dos variables mientras que los modelos multivariantes soportan mayor número de ellas.

En cuanto a la variable gripe solamente se asoció de forma significativa a los ingresos por causas orgánicas en el grupo de mayores de 65 años, con un coeficiente de 0,57 . Por otro lado esta variable, mostró una clara colinealidad con la variable tfrío, de tal manera que, cuando el modelo admitía la variable tfrío en los lags 0 ó 1 , no resultaba significativa la variable gripe y viceversa. Esto resulta lógico si tenemos en cuenta que los episodios de gripe están también asociados con las bajas temperaturas.

Para comparar el grado de ajuste que se alcanza con los modelos multivariantes frente a los univariados, realizamos la representación gráfica simultánea de ambos modelos y la serie real. La figura 7 muestra los dos tipos de modelo frente a la serie de ingresos por enfermedades respiratorias para el total de casos. Tanto en ésta como en el resto de las series, se observa que los modelos multivariantes no difieren en gran medida de los univariados, pero sí se aprecia claramente cómo recogen un mayor número de valores extremos. Con estos modelos sí estamos en condiciones de predecir situaciones episódicas de ingresos que estén relacionados con las variables ambientales analizadas en este trabajo.
Este estudio tiene las limitaciones propias de cualquier estudio ecológico. Además, la utilización de este tipo de datos, basados en la codificación de causas de ingresos, pueden verse afectados por problemas de mala codificación ${ }^{28}$. No obstante, puede suponerse que estos problemas se van a repartir de manera homogénea a lo largo de la serie y lógicamente no deben relacionarse con factores ambientales.

Se puede afirmar, a la vista de los resultados, que las variables ambientales presentan una pequeña pero significativa influencia sobre el número de ingresos por las diferentes causas consideradas. Evidentemente, al incluir en futuros trabajos otras variables ambientales, como la concentración de contaminantes atmosféricos, es de esperar que el efecto del medio ambiente urbano sobre la salud tenga una repercusión aún mayor al descrito en este trabajo, tal como se recoge en la bibliografía sobre el tema ${ }^{27,29-32}$.

Por otro lado, la utilización de este tipo de estudios puede ser una herramienta de gran utilidad en la gestión de los recursos hospitalarios, ya que permite predecir con suficiente antelación posibles excesos en la demanda de este tipo de servicios.

\section{AGRADECIMIENTOS}

A la Unidad de Gestión del Hospital Juan Canalejo así como a la Autoridad Portuaria de A Coruña, por permitirnos disponer, de forma casi inmediata, de los datos requeridos para el estudio.

\section{BIBLIOGRAFÍA}

1. Mackenbach J.P, Kunst A.E., Looman CWN: Seasonal variation in mortality in the Netherlands. J. Epidemiol Comm Health 1992; 46:261-265.

2. Mc Kee C.M. Deaths in winter: can britain learn from Europe? Eur J Epidemiol 1989; 5:178-182.

3. Ballester Díez, F. Meteorología y salud. La relación entre la temperatura ambiental y la mortalidad. Rcv Esp Salud Pública 1996; 70: 251-259. 
4. Pajares S, Díaz J, Montero JC, Alberdi JC, Mirón IJ. Mortalidad diaria en la Comunidad de Madrid durante el periodo 1986-1991 para el grupo de 45 años a 65 años su relación con la temperatura del aire. Rev Esp Salud Pública 1997; 71:149-160.

5. Kunst AE, Looman CWN, Mackenbach JP: Outdoor air temperature and mortality in the Netherlands: A time series analysis. Am J Epidem 1993; 137: 331-41.

6. Alderson MR. Season and mortality. Health Trends 1985;17: 87-86.

7. Kay-Tee Khau. Temperature and cardiovascular Inortality. Lancet 1995; 345: 337-8.

8. Sáez M, Sunyer J, Castellsaguê J, Murillo C, Antó JM. Relationship between Weather temperature and mortality: a time series analisys aproach in Barcelona. Int J Epidemiol 1995; 24 (3): 576-582.

9. Alherdi JC, Díaz, J. Modelización de la mortalidad diaria en la Comunidad de Madrid (19861991). Gac Sanit 1997; 11(1): 7-13.

10. Montero JC, Mirón IJ, Alberdi JC. Díaz J. Influencia de las variables atmosféricas sobre la mortalidad por enfermedades respiratorias y cardiovasculares en los mayores de 65 años en la Comunidad de Madrid. Gac Sanit1997; 11: 164170.

11. Alberdi JC, Díaz J, Montero JC, Mirón IJ. Daily mortality in Madrid Community (Spain) 19861991: Relationship with atmospheric variables. Eur J Epidemiol 1998; 14:571-578.

12. Azevedo E, Ribeiro JA, Lopes F, Martins R, Barros H. Cold: a risk for stroke? J Neurol 1995; 242 (4): 217-21.

13. Anderson HR, Spix C, Medina S, Schouten JP, Castellsagué J, Rossi G, Zmirou D, Touloumi G, Wojtyniak B, Ponka A, Bacharova L, Schwartz J, Katsouyanni K. Air pollution and daily admissions for chronic obstructive pulmonary disease in 6 European cities: results from the APHEA project. Eur Respir J 1997. 19 (5): 1064-71.

14. Tomkinsons A, Bremmer Smith A, Craven C, Roblin DG. Hospital epistaxis admission rate and ambient temperature. Clin Otolaryngol 1995, 20 (3): $239-40$.

15. Tselepidaki IG, Asimakopoulos DN, Katsouyanni K, Moustris C, Touloumi G, Pantazopoulou A. The use of a complex thermohygrometric index in predicting adverse health effects in Athens. Int J Biometeorol 1995; 38(4): 194-8.
16. Schwartz J. Air pollution and hospital admissions for respiratory disease. Epidemiol Resources inc $1995 ; 7: 20-28$.

17. Vigotti MA, Rossi G, Bisanti L, Zanobetti A, Schwartz J. Short term effects of urban air pollution on respiratory health in Milan, Italy 1980 1989. J. Epidemiol Comm Health 1996; 50:71-75.

18. Ramsey JM. The relation of urban atmospheric variables to asthmatic broncho constriction. Bull Environ Contam Toxicol 1976, 16:107-110.

19. Douglas AS, Dunningan MG, Allan TM, Rawles JM. Seasonal variation in coronary heart disease in Scotland. J Epidemiol Comm Health 1995 , 49(6): 575-82.

20. Box GEP, Jenkins G.M., Reimal C. Time series analysis: forecasting and control. Prentice Hall. Englewood Cliffs.1994.

21. Anderson T.W. The statistical analysis of timer series. De. John Wiley; 1971.

22. Díaz J, Alberdi J C, Montero JC, Mirón IJ. Efectos a corto plazo de la contaminación atmosférica sobre la mortalidad en la ciudad de Madrid-España. Información Tecnol1998. 9(3); 33-42.

23. Ballester F, Corella D, Pérez-Hoyos S, Sáez M, Hervás A. Mortality as a function of temperature. A study in Valencia, Spain,1991-1993. Int J Epidemiol 1997.26(3);551-561.

24. Saez M, Sunyer J, Castellsagué J, Murillo C, Antó J M. Relationship between Weather Temperature and Mortality: A Time Series Analysis Approach in Barcelona. Int J Epidemiol 1995. 24(3); 576582.

25. Dorado S, González A, Pérez A, Pereda JM, Alonso F. Influencia de los factores climáticos y de la polución en la agudización de la enfermedad obstructiva crónica pulmonar. Med Clin (Barc) 1982; $78: 226-230$

26. Wen-Harn Pan, Lung-An Li, Ming-Jan Tsai. Temperature extremes and mortality from coronary heart disease and cerebral infarction in elderly chinese. Lancet 1995; 345: 353-355.

27. Díaz J, Alberdi JC, López R, Pajares MS. Ingresos por urgencias en el hospital Gregorio Marañón de Madrid y su relación con factores ambientales. Libro de Ponencias del I Congreso Mundial de Salud y Medio Ambiente Urbano; 1998 6-10 julio. Madrid.

28. Pérez G, Ribalta A, Roset P, Marrugat J, Sala J y los investigadores del grupo Regicor. Fiabilidad del diagnóstico de infarto agudo de miocardio inscrito como causa de muerte en los boletines esta- 
dísticos de defunción en Girona. Gac Sanit 1998;12:71-75.

29. Burnett RT, Dales RE, Raizenne ME, Krewski D, Summers PW, Roberts GR, Raad-Young M, Dann T, Brook J. Effects of low ambient levels of ozone and sulfates on the frequency of respiratory admissions to Ontario hospitals. Environ Res 1994; 65 (2): 172-94.

30. Castellsagué J, Sunyer J, Sáez M, Antó JM. Short-term association of urban air pollution with emergency room visits for asthma. Thorax 1995; 50: 1051-6.

31. Delfino RJ, Becklake MR, Hanley JA. The relationship of urgent hospital admissions for respiratory illnesses to photochemical air pollution levels in Montreal. Environ Res 1994, 67(1): 1-19.

32. Wordley J, Walter S, Ayres JG. Short-term variations in hospital admissions and mortality and particulate air pollution. Occup Environ Med 1997; 54 (2): 108-16. 